\title{
PARTICIPACIÓN BELGA EN EL DESARROLLO DE POLÍTICAS PÚBLICAS EDUCATIVAS DE COLOMBIA EN LA PRIMERA MITAD DEL SIGLO XX
}

\author{
MiRYAM BÁEZ OSORIO \\ Universidad Pedagógica y Tecnológica de Colombia
}

\begin{abstract}
Resumen: Es importante resaltar algunos de los aportes que ofreció el notable maestro europeo Ovide Decroly, quien fuera invitado a Colombia a dictar conferencias sobre sus métodos y aporte pedagógico a maestros de establecimientos educativos, entre ellos a los docentes de la Primera Facultad de Pedagogía que funcionaba en Tunja a fines de la década de los años veinte del siglo XX. Su educación partía del concepto de que la educación era una integración de actividades que permitía a los maestros impulsar centros de aprendizaje y desarrollo para los niños en formación, partiendo del método global de lectura que facilitaba el aprendizaje y la comprensión. Fue así como lo más importante eran los centros de interés que el Ministerio de Educación consideró muy valiosos para la formación de los niños y que realmente estaban en consonancia con las políticas educativas del país. En esas condiciones en la década de los años treinta, el caso de los centros de interés ocuparon la atención de los pedagogos, dado que se interesaron por trabajar con sus educandos aspectos relacionados con la observación, la asociación, las relaciones causa efecto y por supuesto que la expresión cuyos recursos de enseñanza facilitaban los aprendizajes de los niños.
\end{abstract}

Palabras clave: pedagogía, educación, monitor, enseñanza activa.

\begin{abstract}
It is important to highlight some of the contributions offered by the notable European teacher Ovide Decroly who was invited to Colombia to give lectures on his methods and pedagogical contribution to teachers of educational establishments, including teachers of the First Faculty of Pedagogy that worked in Tunja in the late twenties of the twentieth century. His education was based on the concept that education was an integration of activities that allowed teachers to promote learning and development centers for children in training, based on the global method of reading that facilitated learning and understanding. This was how the most important were the centers of interest that the Ministry of Education considered very valuable for the training of children and that were really in line with the country's educational policies. Under these conditions in the thirties, the case of the centers of interest occupied the attention of the pedagogues, since they were interested in working with their students aspects related to observation, association, cause-effect relationships and of course, the expression whose teaching resources facilitated children's learning.
\end{abstract}

Keywords: Pedagogy, Education, Monitor, Active Teaching. 
Participación belga en el desarrollo de políticas públicas educativas de Colombia en la primera mitad del siglo XX

\section{Introducción}

Es importante conocer no solo el pensamiento educativo del maestro belga Ovide Decroly sino sus aportes pedagógicos para la formación de los maestros y maestras de Colombia durante la primera mitad del siglo XX, que sin duda fue de extraordinario valor social y de gran significación a lo largo de aquel siglo en nuestro país y en general en Hispanoamérica.

En el desarrollo de las políticas públicas educativas de Colombia, además de teóricos colombianos, participaron distinguidos educadores y pedagogos de otras nacionalidades tanto de América Latina como de Estados Unidos y de Europa: fue así como se pudo observar el caso del educador Ovide Decroly, quien personalmente estuvo en Colombia hacia la década de los años veinte del mismo siglo XX, dando a conocer sus teorías en educación, que sin duda fueron de impacto, de notable prestigio y aporte significativo.

En esas circunstancias se dieron diferentes acontecimientos y actividades que permitieron el conocimiento de nuevas tendencias pedagógicas y nuevos roles en la enseñanza de los niños de Colombia con la ayuda de teóricos del Viejo Continente y que sin duda prestaron notable ayuda para introducir cambios significativos en la enseñanza de los niños y jóvenes colombianos de aquella época con proyección hacia el siglo XXI.

De ahí la importancia de considerar algunos aspectos que marcaron un hito en la historia educativa y pedagógica del Departamento de Boyacá y Colombia como fueron: la presencia de la Escuela Nueva en nuestro territorio colombiano; la Propuesta Educativa y Pedagógica de Ovide Decroly; Los Centros de Interés Decrolyanos; La Escuela Nueva y sus métodos; Metodologías desde la enseñanza activa como aporte formativo en Colombia; algunas experiencias educativas con el sistema de Ovide Decroly, que sin duda se convirtió en un aporte significativo para la educación durante la época objeto de nuestro estudio.

\section{La escuela nueva en Colombia, un referente de políticas educativas}

La educación colombiana a comienzos del siglo XX comenzó a introducir reformas a finales de la década de los años diez y comienzos de la década de los años veinte, que fue la época cuando el Colegio Gimnasio Moderno de Bogotá se interesó por buscar distintas formas y modelos que permitieran innovar métodos y modelos pedagógicos diferentes que condujeran a introducir cambios notables en el hacer pedagógico y diferentes aspectos de gran significación en el campo educativo.

De esa manera el Establecimiento Educativo denominado Gimnasio Moderno se constituyó en un referente para la orientación de nuevas pedagogías en la enseñanza de Colombia. Aquel Establecimiento educativo dirigido por el notable pedagogo Agustín Nieto Caballero, personaje que tuvo el acierto de contribuir para que unos educadores europeos visitaran el país como el caso del pedagogo Ovide Decroly. Surge así un 
movimiento pedagógico de notable valor histórico y educativo que llevó a transformar métodos en la educación colombiana.

Uno de los líderes de la educación en aquel momento fue Agustín Nieto Caballero, quien desempeñó cargos muy importantes en el sector educativo como rector de la Universidad Nacional. Así mismo, ocupó distintos cargos en diferentes Dependencias en el Ministerio de Educación Nacional y orientó diversos grupos pedagógicos con el fin de encontrar solución a los distintos problemas educativos por los cuales atravesaba el país.

Con aquel ideal educativo y pedagógico se destacan ilustres educadores colombianos como Agustín Nieto Caballero y al ilustre pedagogo antioqueño Tomás Cadavid Restrepo, a quienes se les debe, en buena parte, la iniciación de la Escuela Activa en nuestro país, dentro de las mejores posibilidades que en aquel momento se podía ofrecer para el pueblo colombiano.

En esa circunstancia relacionamos al insigne médico Ovide Decroly (1871-1932), quien por invitación de Don Agustín Nieto Caballero vino a Colombia en el año 1925, y que, siendo un notable pedagogo abnegado, pues solo pidió los gastos de viaje. Dictó conferencias en Bogotá y Medellín sobre la renovación educativa. El ilustre maestro al ver que el Gimnasio Moderno de Bogotá aplicaba sus métodos eficazmente se sorprendió de manera notable. Cautivaron su admiración, lo mismo que la belleza y variedad de los paisajes de Colombia. Consideró que la naturaleza tropical ofrecía admirables elementos educativos a un maestro inteligente y abnegado (Nieto Caballero, s.f.: 193).

\section{Propuesta educativa y pedagógica de Ovide Decroly}

El Maestro Decroly, natural de Renaix -Bélgica-, estudió medicina y se especializó en psiquiatría o tratamiento de niños anormales. En Bruselas fundó en 1901 la Escuela por la vida y para la vida, en la calle de l'Ermitage. Entre sus obras cabe mencionar las siguientes: "La función de la globalización en la enseñanza", "Psicología aplicada a la educación". En colaboración escribió muchas otras, ejemplo: "Hacia la escuela renovada" y "La Práctica de los test mentales". Ovide Decroly consideraba que la escuela tradicional tenía muchos defectos y deficiencias que se debían corregir o enmendar y así consideraba, entre otras cosas:

- Como no se adapta la enseñanza a los intereses evolutivos del niño, era urgente adaptarla.

- Era importante dividir la enseñanza en asignaturas aisladas señalando una hora para aritmética, otra para historia, otra para geografía, etc., las cuales se enseñan aisladamente sin tener en cuenta el interés y se suspenden, para cumplir un horario, a pesar de que esté vivo el interés, de los cuales van saliendo, por natural asociación, las diversas materias.

- No se debe comunicar al niño conocimientos ya elaborados sin su intervención. Debe remediarse atendiendo a los tres procesos naturales que sigue el entendimiento infantil: 
Participación belga en el desarrollo de políticas públicas educativas de Colombia en la primera mitad del siglo XX

observa, asocia y expresa. Luego la enseñanza debe proceder de la observación a la asociación y a la expresión.

- Referente al verbalismo, debe reemplazarse por procedimientos intuitivos, activos y constructivos.

- Los tres procesos de enseñanza que se mencionan a continuación corresponden según Decroly a las ideas asociadas, por tanto, no se suprimían del programa de las asignaturas, sino que se debían tratar en forma distinta:

- Observación. Se iniciaba la lección haciendo que el niño observara y experimentara para que lograra conocimiento personal, no por intermedio del maestro ni de los libros. La observación corresponde a las nociones de ciencias naturales o lecciones de naturaleza. Entraba también en la observación el cálculo mediante comparaciones y medidas.

- Asociación. Este proceso, que es el segundo servía para que el niño conociera aquello que no podía observar ni experimentar, ya porque se encuentra lejos en el espacio ya en el tiempo o sean acontecimientos pasados. En la asociación estaban comprendidas, por consiguiente, la geografía y la historia que se relacionaban con lo observado.

- Expresión. Venía luego la aplicación o expresión de lo aprendido, la cual podía ser en forma concreta: dibujos, modelado, construcción. Así mismo en forma abstracta, por medio de la lectura, la redacción, la conversación (vocabulario). En la expresión quedaban comprendidos el lenguaje, los ejercicios de memoria, el canto y los ejercicios gimnásticos.

- La higiene y la moral, los incluía Decroly en los ejercicios de observación y en los de asociación (Nieto Caballero, 1966: 151-206).

Por otra parte, con relación a la función de la globalización, Decroly recomendaba, no solamente para la enseñanza de la lectura sino también para la didáctica de todas las materias en la escuela elemental, el método global. No partir del elemento separado para ir al todo, sino considerar desde un principio el todo, verbigracia la frase en la lectura en lugar de la letra o sílaba, el lenguaje antes que el análisis gramatical. Se basa Decroly en que la mente infantil no procede por cortes o análisis en la observación, sino que percibe sincréticamente, observa conjuntos vivos, concretos. No los disocia para estudiarlos uno a uno, los aprecia globalmente.

Consecuentemente con su criterio de la enseñanza globalizada, el maestro Decroly empleó en la didáctica de la lectura el método ideo-visual que comienza presentando a los niños, en lugar de letras, frases sencillas que expresan órdenes de fácil cumplimiento y sigue por una serie de ejercicios, a la distinción de palabra, sílaba y letra. Proceso contrario al que anteriormente se seguía. La idea de este método no se debe a Decroly, pues ya desde principios del siglo XIX lo recomendaba Jacotot, pedagogo francés (1770-1840). 


\subsection{El ambiente escolar}

Los espacios de la escuela deben contar con luz suficiente claridad, colores diversos, deben acoger las aulas de la escuela. Bancos comunes, mesas, caballetes, espacio suficiente para una zona verde, paredes tapizadas con dibujos y escritorios de los educandos, mostrándolos ahí como un trofeo. Al igual que se encuentran en las aulas, estantes y armarios con las actividades, producto del trabajo de los educandos.

En la zona verde, si es posible, mantener animales domésticos, de los cuales, los niños debían ser los responsables de brindarles el cuidado necesario. Además, consideraba importante tener en cuenta algunos instrumentos musicales. De la misma manera, se debía colocar en la escuela: lápices, papel, acuarelas, arena para hacer algunas dinámicas, plastilina y otros materiales (Decroly, 1983: 27) consideraba que este método se basaba en los principios de grandes pedagogos de todos los tiempos y que adoptaba también un carácter científico.

Es importante considerar la flexibilidad en métodos por ello se puede afirmar que era el valor de las renovaciones, las experiencias y por ello, hay que comprometerse con la mejora continua, ya que Decroly igualmente pedía que las obras de educación debían ser flexibles, plásticas y capaces de evolucionar.

\subsection{Centros de interés decrolyanos}

Decroly propone los centros de interés entorno de las cuales se agrupan los conocimientos por afinidades o asociaciones. Considera que el descubrimiento de las necesidades del niño permite conocer sus intereses, los cuales atraerán y mantendrán su atención, y así, serán ellos mismos quienes busquen aprender más. En la concepción decrolyana, la observación activa del medio es el método para seguir. En ese sentido, el ambiente escolar era fundamental para la permanencia de los niños en las escuelas.

Podemos observar, la flexibilidad en cuanto al método, por ello es por lo que podemos afirmar que era muy importante para él, el valor de las renovaciones, las experiencias y por ello, había que comprometerse con la mejora continua, ya que Decroly igualmente pedía que las obras de educación debían ser flexibles, plásticas y capaces de evolucionar. Así mismo, un centro de interés es la síntesis entre las exigencias del respeto a las aspiraciones propias del niño y las presiones de la formación intelectual. El centro de interés se organiza a partir de las siguientes fases:

Observación como punto de partida de las actividades intelectuales y base de todos los ejercicios, debe ser continua y debe llevarse a cabo en el medio natural. Asociación: a) de las dimensiones espaciales b) de las dimensiones temporales lejanas c) asociaciones tecnológicas (empleo de materias primas, adecuación al medio) d) relaciones de causa y efecto. Expresión, abarca todo aquello que permita la manifestación del pensamiento de modo accesible a los 
Participación belga en el desarrollo de políticas públicas educativas de Colombia en la primera mitad del siglo XX

demás. La enseñanza debía organizarse de acuerdo a estas consideraciones, así el alumno ejercería de manera activa, sus capacidades intelectuales para adaptarse felizmente a su ambiente humano y físico (Bohórquez Casallas, 1956: 438).

Todos los centros de interés se basan en los intereses del niño, vistos según el criterio decrolyano (intereses naturales). El niño es el centro de toda la enseñanza: paidocentrismo. El programa de centros busca dos fines: que el niño se conozca así mismo y que conozca el ambiente en que vive, comprende, por tanto: sus necesidades; alimentación; defensa contra la intemperie (vestido, vivienda); protección contra los peligros y accidentes; necesidad del trabajo; medio; familia; escuela; sociedad; animales; vegetales; minerales (tierra, aire, agua etc.) y el sol, la luna y las estrellas.

Allí, en las estrellas, se detiene la pedagogía decrolyana, carece de Dios, es simplemente una pedagogía naturalista que es, por los campos de la materia, pues se abstiene de considerar alguna referencia del Creador que para algunos no debía ser. Lo común en aquella época era que se tuviera en cuenta la protección de Dios para los niños.

Grandes son, sin duda, los méritos del pedagogo belga. Su amor a la niñez, los novedosos procedimientos con que ha enriquecido la didáctica, todo ello le acredita como una figura ilustre en la historia de la pedagogía. Su bondad, la dulzura de su carácter, su moralidad acrisolada, su vida limpia, le merecieron ser llamado "santo laico". Empero no hay que tomar todo lo suyo a ciegas ni seguirlo confiadamente. Se ha considerado, por ejemplo, que los centros de interés decrolyanos, por estar sometidos a los intereses y deseos del niño, resultan dispersos, derraman el conocimiento, restándole unidad y concentración.

Así mismo, respecto a la formación del carácter moral, que es la más elevada tarea de la educación, exige la educación de la voluntad y esta es precisamente la facultad que deja huérfana la escuela decrolyana por atender directamente a las apetencias del educando, sin exigirles conformarlas a un código de valores superiores, sin exigirle sacrificar el interés cuando es contrario al deber. La escuela de la vida que construye Decroly olvida y teme el problema de la muerte. No basta la acción orgánica, vivir sensiblemente, hay que aprender a morir, a mortificarse para no ahogar el espíritu.

Por otra parte, el naturalismo de Ovide Decroly era particular, presenta ausencia de Dios en la educación. Por esa razón algunos teóricos consideraban que era funesta la educación orientada con los postulados de Ovide Decroly, pues en el caso particular de Colombia no era bienvenido, puesto que la orientación naturalista de la escuela tenía carácter laico. En sus centros de interés se notaba la ausencia de la vida sobrenatural. No encontró Decroly más intereses educativos para el niño que el alimento, el abrigo, los animales y cosas materiales. Algunos criticaron a Decroly porque había desterrado de la escuela el catecismo, es decir que había prescindido del fin supremo del niño. Al colocar Decroly al niño como centro de la vida y de la verdad, olvidó que Cristo es el camino, es la verdad y la vida, abandonó al niño a las miserias de su naturaleza, olvidando que había un Ser Supremo. 
Miryam Báez Osorio

\section{Ovide Decroly y la escuela nueva en el Gimnasio Moderno de Bogotá}

Los colaboradores de la revista Cultura no solo deseaban una reforma de la estructura de la instrucción pública sino una reforma profunda de los métodos de enseñanza en escuelas y colegios. En 1925, Agustín Nieto Caballero respondió a esos deseos de invitar a Ovide Decroly a una visita al Gimnasio Moderno, para exponer sus concepciones sobre la Escuela Nueva. Anticlerical notorio, el médico belga había trabajado primero en neurología, después se especializó en la educación de niños anormales para los cuales fundó un instituto, y más tarde se orientó hacia la educación general.

Para Helg, según Decroly, el mayor error de los métodos tradicionales consistía en considerar a los niños como adultos en miniatura y no como niños integrales; por ello recurrían a la memorización y a una disciplina excesiva que volvía pasivos a los alumnos. Planteó, como se señaló anteriormente, una pedagogía en tres etapas: la observación por el niño de la realidad y su contorno; la asociación entre estas observaciones y la teoría y la expresión tanto oral como escrita o manual de los conocimientos adquiridos a partir de las dos primeras fases. Centros de interés orientados hacia las actividades esenciales del hombre como: la alimentación, el trabajo, el vestido, por ejemplo, permitían concretar esta pedagogía. Finalmente, mediante el método de la globalización, la enseñanza estimulaba el interés de los alumnos hacia materias más escolares (Helg, 1987: 121).

Las conferencias de Decroly en el Gimnasio Moderno atrajeron a los pedagogos de todo el país. Originaron los primeros centros de interés en algunas escuelas privadas, antes de la adopción oficial del método en 1936. Confirmaron sobre todo el papel que como centro pedagógico desempeñó durante mucho tiempo el Gimnasio Moderno para el país.

Con Decroly vinieron un médico belga, Pierron y un profesor de pedagogía de la Universidad Católica de Lovaina, Raymond Buyse. Los tres permanecieron dos meses, principalmente en Bogotá, sirviendo como asesores al Gimnasio Moderno; otros colegios recibieron también su visita. Los hermanos cristianos tuvieron íntimos contactos con esta misión oficiosa y uno de ellos recibió una beca del gobierno belga para realizar un doctorado en pedagogía bajo la dirección de Buyse. Decroly visitó igualmente escuelas de Boyacá, donde desde fines de 1924 el pedagogo conservador Rafael Bernal Jiménez, sobrino de Miguel Jiménez López, había emprendido una reforma de la educación pública.

Señalamos estas correlaciones con los sectores conservadores moderados y con una comunidad religiosa porque ilustran la división de los católicos acerca de la reforma educativa. En efecto, el episcopado se opuso a los métodos pedagógicos de Decroly porque este era abiertamente anticlerical. Cuando el ministro conservador Julio Carrizosa Valenzuela publicó en 1932 un folleto sobre el médico belga, el arzobispo de Bogotá, Ismael Perdomo, vetó su distribución, pues Decroly defendía, según él, el monopolio de la educación por el Estado, la coeducación de los sexos y la tolerancia religiosa en la escuela; además, Decroly daba una definición errónea de la religión (Helg, 1987: 122).

Acta Hispanica, Hungría, Supplementum II: 817-827, 2020, ISSN: 1416-7263 | 823 
Participación belga en el desarrollo de políticas públicas educativas de Colombia en la primera mitad del siglo XX

\section{Experiencia educativa del Departamento de Boyacá con influencia belga}

En el Departamento de Boyacá como en otras regiones de Colombia, respecto a la enseñanza primaria, obedeciendo a los postulados del pedagogo Ovide Decroly, se le dio especial atención a la higiene escolar, a pesar de que Boyacá era una de las regiones más pobres de Colombia y sus tasas de analfabetismo y ausentismo escolar eran particularmente elevadas.

Por esa razón desde el nombramiento en el cargo de Director de Instrucción Pública del departamento, Rafael Bernal Jiménez, en el año 1925 se propuso mejorar notablemente la educación en su región natal. El personal de la Dirección de Instrucción Pública se dedicó a realizar estudios con los maestros de la región y a visitar las escuelas primarias. Allí lograron detectar dos problemas: la pobreza y la debilidad física de los alumnos, por una parte y por otra, la falta de formación en los profesores.

Para remediar esto, Bernal Jiménez propuso un plan de reorganización de la instrucción pública. Logró poderes extraordinarios en la Asamblea de Boyacá, así como el apoyo del gobernador Silvino Rodríguez, quien era amante de las cuestiones educativas. De ahí que, más adelante va a ejercer el cargo de Ministro de Educación durante el año 1926.

La reforma exigía un presupuesto menos estrecho para la instrucción pública, pero Boyacá era el departamento que asignaba el porcentaje más bajo a la educación, aunque desde 1927 aumentó considerablemente al pasar de 91.000 pesos en 1926 a 173.942 pesos. El ingreso destinado a la educación fue entregado en adelante a la dirección departamental de instrucción pública. Así se puso fin a la descentralización financiera y se mejoró un poco la situación en el campo educativo.

Desde el comienzo de la administración, el educador Bernal Jiménez buscó también recursos financieros independientes. Tuvo la idea de crear sociedades comunales de patronato escolar, compuestas de damas y notables de las aldeas. Mediante bazares, ventas de caridad y colectas, las sociedades de patronato debían hacer participar a la comunidad en el sostenimiento de la escuela y asegurar cierta redistribución de bienes en la aldea (Helg, 1987: 123).

Otro aspecto de singular importancia fue la formación de Bibliotecas, así algunos municipios lograron formar una pequeña biblioteca para la escuela, gracias a donaciones de libros y otras actividades. Otras personas donaban vestidos a los niños. Así mismo, el dinero recolectado servía para financiar restaurantes o cantinas escolares en los cuales los estudiantes podían tomar algunos alimentos especialmente para aquellos que vivían lejos de la escuela. En 1927 cerca de 2.000 niños de los 34.182 matriculados en las escuelas primarias públicas del departamento de Boyacá se beneficiaron de aquellos restaurantes escolares (Secretaría de Educación, 1927: 10).

Por otra parte, en la línea de la campaña de higiene recomendada por los intelectuales, en el departamento de Boyacá se fundó el primer servicio de medicina escolar que fue 
financiado gracias a la supresión de los puestos de policía escolar. A pesar de esto el servicio fue difícil de organizar. Los cinco médicos contratados al principio no llegaban a visitar todas las escuelas y sus bajos salarios no les permitían estar bien equipados; además debían desempeñar también las funciones de médicos legistas del departamento. Pero en 1933 los médicos escolares no se ocupaban ya sino de las escuelas y el departamento les suministraba medicamentos; no solamente podían organizar una campaña sanitaria, sino que al año siguiente se estableció también un servicio de medicina legal (Secretaría de Educación, 1932-1933: 8-15).

Además, Rafael Bernal Jiménez favoreció las iniciativas particulares de los maestros y algunos comenzaron a aplicar los métodos de la Escuela Nueva, es el caso de Norberto Solano Lozano quien había hecho de su escuela urbana una pequeña cooperativa de producción provista de una granja cuyos productos se destinaban al restaurante escolar. La dirección de instrucción pública impulsó a los profesores a dar paseos con su clase y a dictarles las lecciones al aire libre y prohibió los castigos corporales y el empleo de la férula.

Ciertamente los seis años de la administración de Rafael Bernal Jiménez no fueron suficientes para enderezar la situación educativa y sanitaria de Boyacá. Solo una minoría de escolares - con frecuencia pertenecientes a los pueblos y no a las veredas - se benefició de los restaurantes escolares y del servicio médico escolar. Los niños necesitados, los que ni siquiera podían asistir a la escuela, continuaron ignorados. A pesar de todo, el progreso escolar del departamento fue notable: entre 1927 y 1932, el número de escuelas pasó de 614 a 784, o sea un aumento del 27.7\%, mientras que en el resto del país seguía igual.

Sobre todo, esta reforma, sirvió de laboratorio y modelo a las reformas ulteriores. Dejó además percibir las influencias de la misión pedagógica alemana, de la Escuela Nueva y de Miguel Jiménez López. Después de Boyacá, el Tolima conoció una experiencia similar. En 1930, el liberal Antonio Rocha fue nombrado gobernador del Tolima y con el fin de desarrollar la educación, escogió como Director de Instrucción Pública a Arcadio Dulcey, originario de Boyacá y colaborador de Bernal; Dulcey se rodeó de un grupo de jóvenes inspectores que organizaron, cada uno en su zona, sociedades de patronato escolar, servicio médico y restaurantes escolares y buscaron interesar a los maestros en la nueva pedagogía (Secretaría de Educación, 1928: 60).

\section{Conclusiones}

Los esfuerzos de algunos dirigentes colombianos en el campo educativo y pedagógico hacia la década de los años veinte del mismo siglo veinte los llevaron a considerar en la necesidad de traer misiones pedagógicas que orientaran la educación hacia un mejoramiento de la enseñanza escolar en diversos niveles. Sin embargo, fue prioritario velar por la enseñanza en diversos niveles de formación, especialmente de la escuela elemental y primaria.

Así mismo en atención a diversas situaciones, se señala al maestro Decroly como a uno de los mayores pedagogos que llegó a Colombia a dar a conocer sus métodos y 
Participación belga en el desarrollo de políticas públicas educativas de Colombia en la primera mitad del siglo XX

orientaciones generales para el mejoramiento de la educación. Así el médico y pedagogo Ovide Decroly, oriundo de Bélgica y quien se ingenió la enseñanza global y los Centros de Interés fue el maestro por excelencia que vino a orientar un nuevo modelo de educación que él mismo creó.

De acuerdo con el método traído por Ovide Decroly, la enseñanza debía organizarse en proporción a ciertos ambientes y consideraciones del contexto, así el alumno ejercería de manera activa sus capacidades intelectuales para adaptarse felizmente a su ambiente humano y físico, en concordancia con el contexto.

Con respecto al espacio y recursos de aprendizaje, el maestro y los dirigentes educativos debían tener presente aspectos de contexto, entre otras cuestiones: la luz, es decir que el niño tuviera claridad que contara con colores diversos y en general las aulas de clase fueran lo mejor para sus aprendizajes en cualquier escuela. Además, contar con bancos comunes, mesas, caballetes, espacio suficiente para una zona verde, como mínimo. Al igual que se encontrarán en las aulas, estantes y armarios propios para las actividades, producto del trabajo de los educandos.

De igual manera, el pedagogo Decroly consideraba de suma importancia, si era posible, mantener animales domésticos, en donde los niños pudieran ser los responsables de brindarles el cuidado necesario, para que no solo conocieran los contextos y la importancia de su cuidado, sino que en el futuro fueran capaces de asumir el ejercicio como tal.

Así mismo dentro del método decrolyano, se consideraba importante tomar en cuenta algunos instrumentos musicales para que los niños pudieran abrir el espacio de la formación musical que según el pedagogo traía muchos beneficios en la educación de los niños y niñas, asunto importante porque era poder contar con otras posibilidades en el medio para la formación de los educandos.

Los postulados de Decroly llamaron la atención de todos los pedagogos del país, pero las dificultades económicas para atender la educación rechazaron algunas ideas. Por eso en principio solo se originaron los primeros centros de interés en algunas escuelas privadas y no en las oficiales, aunque más adelante y solo hacia el año 1936, se adopta el método decrolyano.

Con las enseñanzas de dicho pedagogo, la educación colombiana cambió de manera notable, dirigentes, maestros y sociedad en general vieron la necesidad de dar un vuelco al tema educativo y así se logró integrar procesos y ayudar a mejorar la educación del país, haciendo énfasis en los cambios de metodologías en la enseñanza como el caso de los centros de interés, pensando siempre en los fines de la educación que como decía Decroly debía ser la búsqueda del desarrollo y la conservación de la vida.

Así mismo, la pedagogía decrolyana mostraba que el niño era un ser capaz de comprender la realidad y como tal sus aprendizajes eran fáciles y de gran beneficio para las comunidades y la sociedad en general, pues los alumnos debían aprender de la realidad tal cual era, pues los intereses de cada uno nacían o aparecían de su propia realidad. 
Miryam Báez Osorio

\section{Referencias bibliográficas}

Bohórquez Casallas, Luis Antonio (1956). La evolución educativa en Colombia. Bogotá: Publicaciones Cultural Colombiana.

Decroly, Ovide - Monchamp, E. (1983). El juego educativo. Iniciación a la actividad intelectual y motriz: Madrid: Ediciones Morata, S.L.

Helg, Aline (1987). La educación en Colombia 1918-1957. Una historia social, económica y política. Bogotá: CEREC.

Nieto Caballero, Agustín (1966). Una escuela. Bogotá: Antares-Tercer Mundo S.A.

Nieto Caballero, Agustín (s.f.). Escuela Activa. Selección de textos. Bogotá: Presencia.

Secretaría de Educación de Boyacá (1920-1950). Informes Secretarios de Educación. Biblioteca del Departamento de Boyacá, Archivo Departamental de Boyacá. 